\title{
Article \\ Preferred Limb Reaction, Swing and Recovery Step Times between Subjects with and without Chronic Low Back Pain
}

\author{
Paul S. Sung ${ }^{1, *}$ and Emily Hosmer ${ }^{2}$ \\ 1 Department of Physical Therapy, Indiana Wesleyan University, Marion, IN 46953, USA \\ 2 Department of Health Sciences, Central Michigan University, Mount Pleasant, MI 48859, USA; \\ hosme1ee@cmich.edu \\ * Correspondence: paul.sung@indwes.edu
}

check for updates

Citation: Sung, P.S.; Hosmer, E. Preferred Limb Reaction, Swing and Recovery Step Times between Subjects with and without Chronic Low Back Pain. Symmetry 2021, 13, 2115. https://doi.org/10.3390/ sym 13112115

Academic Editor: John H. Graham

Received: 17 September 2021

Accepted: 25 October 2021

Published: 8 November 2021

Publisher's Note: MDPI stays neutral with regard to jurisdictional claims in published maps and institutional affiliations.

Copyright: (c) 2021 by the authors. Licensee MDPI, Basel, Switzerland. This article is an open access article distributed under the terms and conditions of the Creative Commons Attribution (CC BY) license (https:// creativecommons.org/licenses/by/ $4.0 /)$.

\begin{abstract}
A compensatory stepping strategy following repeated perturbations may compromise dynamic balance and postural stability. However, there is a lack of study on preferred limb reaction, swing, and step time adjustments. The purpose of this study was to investigate limb reaction, swing, and recovery step times following repeated trip perturbations in individuals with and without non-specific chronic low back pain (LBP). There were 30 subjects with LBP and 50 control subjects who participated in the study. The limb reaction, swing, and recovery step times (s) were measured following treadmill-induced random repeated perturbations $(0.12 \mathrm{~m} / \mathrm{s}$ velocity for $62.5 \mathrm{~cm}$ displacement), which caused subjects to move forward for $4.90 \mathrm{~s}$. Both groups demonstrated a significant interaction of repetitions and times $(F=4.39, p=0.03)$. Specifically, the recovery step time was significantly shorter in the LBP group during the first trip $(\mathrm{t}=2.23, p=0.03)$. There was a significant interaction on repetitions and times $(\mathrm{F}=6.03, p=0.02)$ in the LBP group, and the times were significantly different $(\mathrm{F}=45.04, p=0.001)$. The initial limb reaction time of the LBP group was significantly correlated with three repeated swing times to avoid falls. The novelty of the first trip tends to enhance a protective strategy implemented by the LBP group. Although limb preference did not demonstrate a significant difference between groups, the LBP group demonstrated shorter recovery step times on their preferred limb initially in order to implement an adaptive strategy to avoid fall injuries following repeated perturbations.
\end{abstract}

Keywords: reaction time; recovery step time; perturbations; low back pain; compensation; balance

\section{Introduction}

Low back pain (LBP) affects up to $80 \%$ of the population, and those who have nonspecific chronic LBP exhibit neural changes that underlie balance deficits, which may be related to postural control [1-3]. However, there is a lack of understanding on preferred lower limb reactions to avoid fall injuries as well as recovery step responses to postural adjustments.

Individuals with LBP compensate their lower limb reactions to avoid further injuries or pain recurrences. Recent studies summarized a central idea that foot placement is important in implementing adaptive dynamic balance for gait stability [4-6]. The foot placement and proper timing provided explicit predictions on where to place the foot relative to the body at each step as well as the active compensation for center of mass $(\mathrm{COM})$ and center of pressure (COP) during the preceding steps. The altered patterns of limb reaction, swing, and recovery step times might lead to the adoption of compromised dynamic balance strategies from motor deficits in individuals with LBP. However, little is known about adaptive lower limb control preference when considering swing and recovery step times following repeated trip perturbations.

Individuals with LBP demonstrate reduced trunk stability and compensated responses to higher loading while perturbations were provided by the treadmill [7]; a perturbation with a handheld task (so-called dual-task walking) can commonly represent coordinated 
activities of daily living [8]. These results are clinically relevant since individuals with LBP possess altered trunk control and somatosensory dysfunction [3,9]. For instance, those who suffer with LBP demonstrate a trunk strategy and rely more on ankle proprioception to control their upright standing [10]. Asymmetric gait in LBP groups is often unstable, and maintaining upright balance requires dynamic control. The adaptive strategies on lower limb reactions and foot placement are critical when considering limb preference between right limb dominant individuals with and without LBP.

In addition, other studies compared gait speed; however, the gait stability based on swing and recovery step times relative to limb reactions would be critical to control dynamic balance. It was evident that the shorter stance phase enables the possibility of a stretch reflex to contribute to a strong contraction during push-off [11]. The functional relationship between limb reactions and ankle response times could lead to a better understanding of fall prevention strategies. It would be valuable for clinicians to clarify the mechanism of trunk-ankle integration as it relates to postural stabilization, using similar characteristics of individuals with and without LBP. It is also important to consider confounding factors, such as age and body mass index (BMI), to ensure the generalizability of the results. It might be expected that LBP subjects compensate their recovery step time in response to first trip perturbations and adjust with repeated perturbations in order to prevent fall injuries.

Therefore, the aim of our study was to investigate preferred limb reaction, swing, and recovery step times following repeated trips between individuals with and without LBP. Our first hypothesis was that individuals with LBP would demonstrate longer limb reaction and recovery step times following repeated trip perturbations. Our second hypothesis was that the limb reaction time delays were associated with the swing times following the first trip in the LBP group.

\section{Materials and Methods}

\subsection{Subjects}

Subjects were recruited from the community through local advertisements and fliers, and those subjects who met the study's inclusion criteria signed the University Institutional Review Board approved informed consent form. Subjects were eligible to participate if they had no current episode of acute pain referral into the lower limbs and had continued presence of back pain for more than 3 months.

Subjects were excluded from participation if they: (1) had a diagnosed psychological illness (such as depression or schizophrenia) that might interfere with the study protocol [12], (2) had overt neurological signs (sensory deficits or motor paralysis), and/or (3) were pregnant. The subjects in our study were all right limb dominant, and the right lower limb was determined as the dominant side for all subjects [13]. Right-handedness was confirmed using the Edinburgh Handedness Inventory for skilled motor tasks [14]. The control group was recruited based on the demographic factors of the LBP group.

\subsection{Experimental Procedures}

Upon arrival to the Motion Analysis Center, each subject completed a health screening form. The level of disability related to LBP for all subjects was assessed by the Oswestry Disability Index (ODI) [15], since it has been a valuable outcome measure with high validity and reliability $[16,17]$.

Following the instructions, the subjects walked for one minute without perturbation on the ActiveStep ${ }^{\circledR}$ treadmill (Simbex, Lebanon, NH, USA) to be familiar with the device. They were instructed that they may experience a trip perturbation. For the first three trials, the subjects held a weighted tray (approximately $1 \mathrm{~kg}$ ) with an empty cup on it. If the perturbation occurred, subjects tried to correct their balance while still holding the tray in their hands. Following the first trial, the subjects were expected to recover a standing position. At no time were the subjects informed of when, where, or how the trip would occur. Additionally, all subjects wore a full-body safety harness that imposed 
negligible resistance, but still protected them from any potential injuries should they lose their dynamic balance.

The ActiveStep ${ }^{\circledR}$ treadmill has a two-ply belt, and the belt was free to slide forward on top of a low-friction metal frame embedded in the treadmill. Perturbations were designed to mimic a trip hazard that subjects might experience in their daily life, and the treadmillinduced random repeated perturbations $(0.12 \mathrm{~m} / \mathrm{s}$ velocity for $62.5 \mathrm{~cm}$ displacement $)$ caused them to move forward for $4.90 \mathrm{~s}$ (Figure 1). The limb reaction, swing, and recovery step times were analyzed based on the $\mathrm{x}$ and $\mathrm{y}$ coordinates at a $60 \mathrm{~Hz}$ sampling rate from the pressure mat installed beneath the platform (GAITRite, Sparta, NJ, USA). As the leading foot touched down during a recovery step by random perturbation, a computer-controlled mechanism would release the movable belt on the treadmill and allow the subject to move forward. The data points were synchronized to produce recovery swing and step times.

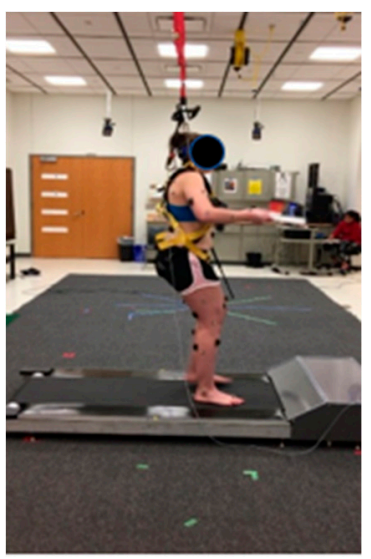

(A)

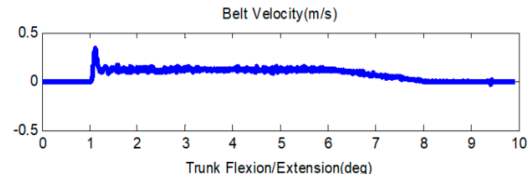

Trunk Flexion/Extension(deg)
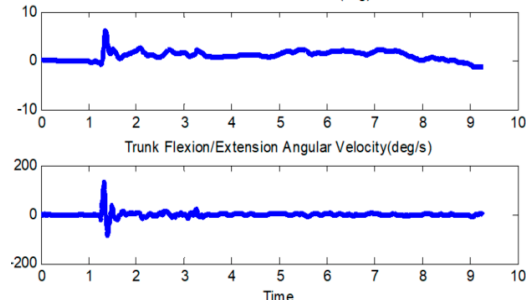

(B)

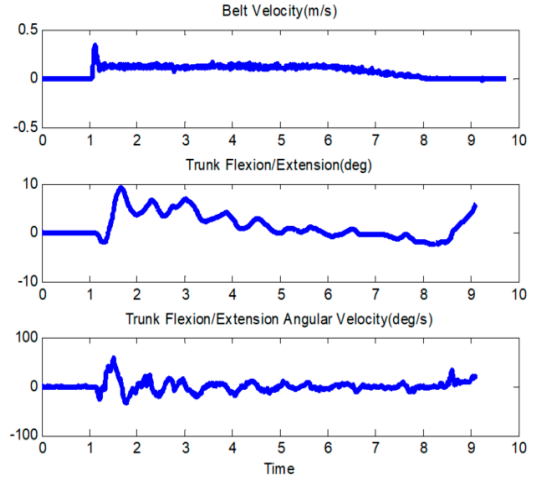

(C)

Figure 1. (A) The experimental protocol used a treadmill to produce trip perturbations in standing while holding a tray. The perturbation was provided at $0.12 \mathrm{~m} / \mathrm{s}$ velocity for $62.5 \mathrm{~cm}$ displacement, which caused subjects to move forward for $4.90 \mathrm{~s}$. (B) An example of angle and velocity changes while holding a tray following the perturbation for a control subject. (C) The subject with low back pain (LBP) demonstrated greater angular displacement and velocity changes.

The inertial measurement unit (IMU, ActiveStep ${ }^{\circledR}$ treadmill) is a device that measures the acceleration vectors and consists of a triaxial accelerometer and a gyroscope. The IMU was attached to the back of the subject's harness using a clip, and the orientation of the IMU was parallel to the fourth thoracic spine of the subject. The kinematic changes on angle, as well as velocity, were measured by the sensor during the entire profile.

Handheld tasks (so-called dual-task walking) represent functional activities of daily living [8]. The perturbation protocol utilized the functional trials from previous studies $[18,19]$ and was applied during walking on a treadmill while random perturbations were provided [7]. In order to improve validity, the subjects in our study were only right limb dominant. The following dependent variables measured the responses of the first step after perturbations:

- $\quad$ Limb reaction time (s) was determined as the time between onset of the treadmill motion and recovery of limb motion before toe-off following a trip.

- Swing time (s) was initiated with toe-off and ended with initial contact of the same foot.

- Recovery step time (s) was defined as the time delay from the initial contact of one foot to the opposite foot.

\subsection{Statistical Analysis}

Statistical analyses were completed using IBM Statistics 22. Normality was assessed for the dependent variables (limb reaction, swing, and recovery step times) by the Shapiro -Wilks test. For preliminary power analyses associated with limb reaction time measures, we estimated variance in means between groups is 9.67 , the estimated variance in means 
between levels is 17.12 , and the estimated variance in means among levels by groups is 3.55. Furthermore, the between-groups error term is 9.40 , the within-group error term is 8.27 , the measure of sphericity is 0.91 , and the bias term multiplier is -1.07 . With the test significance level $=0.05$ and between groups power $=99 \%$, between levels power $=99 \%$ and levels by groups power $=80 \%$, we needed 54 subjects (with and without LBP) based on the method by Muller and Barton [20].

An independent $t$-test was also conducted to compare group differences. The mixed repeated measure analysis of variance (ANOVA) was conducted between groups. The independent variables were trials based on three repeated perturbations (repetitions); reaction, swing, and step times (times); and groups with and without LBP (groups). The individual factors, such as age and BMI, were used as covariates in the analysis if a group difference was confirmed. A Pearson correlation analysis was used to analyze the degree of associations between groups for the dependent variables. For all statistical tests, the type I error rate was set at 0.05 .

\section{Results}

In total, 30 subjects with LBP and 50 control subjects participated in the study (Chi-square $=2.64, p=0.10$ ). There was no significant age difference between groups ( $25.68 \pm 8.65$ years old in the control group vs. $30.30 \pm 13.53$ years old in the LBP group, $95 \%$ confidence interval $[-9.55 / 0.31] ; \mathrm{t}=-1.86, p=0.07)$. The BMI was not significantly different between groups ( $23.71 \pm 4.54$ in the control group vs. $25.41 \pm 5.81$ in the LBP group, $95 \%$ confidence interval $[-4.02 / 0.62] ; \mathrm{t}=-1.45, p=0.15)$. The level of disability based on the ODI was $21.07 \pm 9.06 \%$, which indicated a moderate disability level.

As shown in Table 1, the limb preference difference was analyzed between groups for three repeated perturbations. At the first perturbation, both groups used the dominant limb for the first step $(31 / 50=62 \%$ for the control group vs. $17 / 30=57 \%$ for the LBP group; Chi-square $=0.22, p=0.64)$, the second step $(28 / 50=56 \%$ for the control group vs. $16 / 30=53 \%$ for the LBP group; Chi-square $=0.06, p=0.81)$, and the third step $(32 / 50=64 \%$ for the control group vs. $18 / 30=60 \%$ for the LBP group; Chi-square $=0.13, p=0.72$ ). Overall, there was no significant limb difference between groups.

Table 1. Limb preference for the recovery step following repeated perturbations between groups.

\begin{tabular}{|c|c|c|c|c|c|}
\hline & & Control Group & LBP Group & Chi-Square & $p$ \\
\hline \multirow{2}{*}{ Perturbation 1} & Dominant & $31(62.0 \%)$ & $17(56.6 \%)$ & \multirow{2}{*}{0.22} & \multirow{2}{*}{0.64} \\
\hline & Non-dominant & $19(38.0 \%)$ & $13(43.4 \%)$ & & \\
\hline \multirow{2}{*}{ Perturbation 2} & Dominant & $28(56.0 \%)$ & $16(53.3 \%)$ & \multirow{2}{*}{0.06} & \multirow{2}{*}{0.81} \\
\hline & Non-dominant & $22(44.0 \%)$ & $14(46.7 \%)$ & & \\
\hline \multirow{2}{*}{ Perturbation 3} & Dominant & $32(64.0 \%)$ & $18(60.0 \%)$ & \multirow{2}{*}{0.13} & \multirow{2}{*}{0.72} \\
\hline & Non-dominant & $18(36.0 \%)$ & $12(40.0 \%)$ & & \\
\hline
\end{tabular}

LBP: low back pain.

The correlation analyses were conducted to compare the limb reaction times with swing and step times following repeated perturbations based on the first limb reaction times (Table 2). At the first perturbation, the reaction and swing times were significantly correlated in both groups. At the second perturbation, the limb reaction time was correlated with the first reaction time $(r=0.62, p=0.001)$ in the control group. The LBP group demonstrated significant correlations with first reaction time $(r=0.81, p=0.001)$ and swing time $(\mathrm{r}=0.67, p=0.001)$. At the third perturbation, the limb reaction times were correlated with the first reaction time $(\mathrm{r}=0.53, p=0.001)$ and swing time $(\mathrm{r}=0.37, p=0.01)$ in the control group. In the LBP group, the reaction times $(r=0.41, p=0.03)$ and swing time $(\mathrm{r}=0.41, p=0.03)$ were positively correlated with the first reaction time. 
Table 2. Correlation of first limb reaction times with other measures following perturbations between groups.

\begin{tabular}{llll}
\hline & & \multicolumn{1}{c}{ Control Group } & \multicolumn{1}{c}{ LBP Group } \\
\hline \multirow{2}{*}{ Trial 1 } & Reaction time & 1.00 & 1.00 \\
\cline { 2 - 4 } & Swing time & $0.57(0.001)^{*}$ & $0.56(0.001)^{* *}$ \\
\cline { 2 - 4 } Trial 2 & Step time & $0.18(0.19)$ & $0.28(0.13)$ \\
\hline \multirow{3}{*}{ Trial 3 } & Reaction time & $0.62(0.001)^{* *}$ & $0.81(0.001)^{* *}$ \\
\cline { 2 - 4 } & Swing time & $0.23(0.10)$ & $0.67(0.001)^{* *}$ \\
\cline { 2 - 4 } & Step time & $0.21(0.14)$ & $0.18(0.34)$ \\
\hline & Reaction time & $0.53(0.001)^{* *}$ & $0.41(0.03)^{*}$ \\
\cline { 2 - 4 } & Swing time & $0.37(0.01)^{*}$ & $0.41(0.03)^{*}$ \\
\cline { 2 - 4 } & Step time & $0.21(0.13)$ & $0.16(0.38)$ \\
\hline
\end{tabular}

Pearson $\mathrm{r}(p)$, LBP: low back pain, ${ }^{*} p<0.05,{ }^{* *} p<0.01$.

As shown in Figure 2, a mixed repeated measure ANOVA was conducted on limb reaction, swing, and step times between groups following three repeated perturbations. The main effect of repetitions $(\mathrm{F}=21.69, p=0.001)$ and times $(\mathrm{F}=139.47, p=0.001)$ was significantly different between groups; and the results of interactions were significant between repetitions and times $(\mathrm{F}=10.69, p=0.002)$. More importantly, the groups demonstrated a significant interaction on repetitions and times $(\mathrm{F}=4.39, p=0.03)$. Specifically, the recovery step time was significantly shorter in the LBP group at the first perturbation $(t=2.23, p=0.03)$.

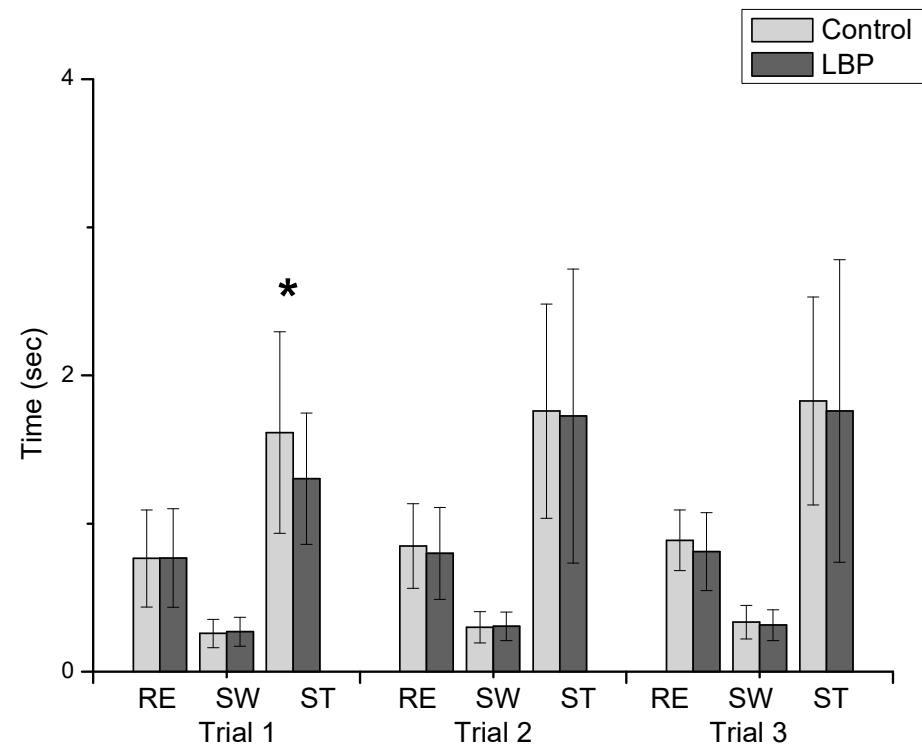

Figure 2. Results of repeated measure ANOVA on limb reaction, swing, and step times between groups following three repeated perturbations. The groups demonstrated a significant interaction on repetitions and times $(\mathrm{F}=4.39, p=0.03)$. RE: reaction time, $\mathrm{SW}$ : swing time, ST: step time, the bar indicates standard deviation, ${ }^{*} p<0.05$.

In Figure 3, the limb preference for those dependent variables was compared between groups. The control group demonstrated significant differences on repetitions $(\mathrm{F}=24.06$, $p=0.001)$ and reaction times $(\mathrm{F}=97.04, p=0.001)$; however, there was no interaction between repetitions and reaction times $(\mathrm{F}=0.82, p=0.37)$. The groups demonstrated a significant difference on reaction times $(\mathrm{F}=45.04, p=0.001)$ as well as a significant interaction on repetitions and reaction times $(\mathrm{F}=6.03, p=0.02)$, although there was no difference in repetitions $(\mathrm{F}=3.09, p=0.09)$. 


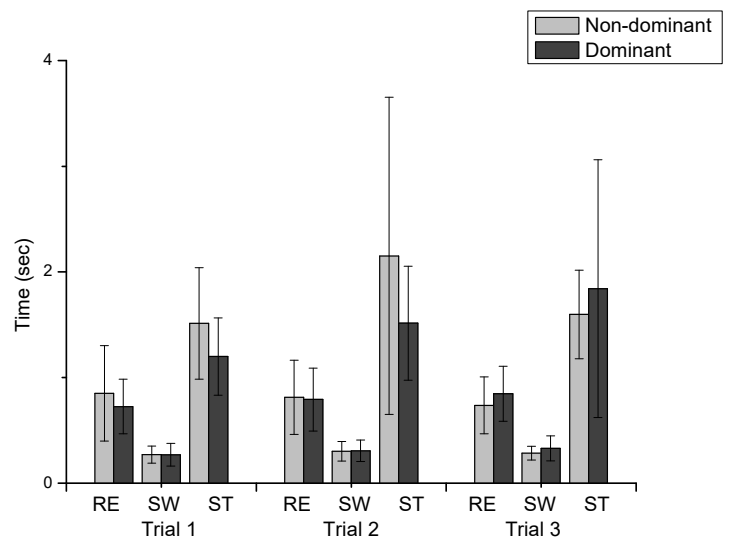

(A)

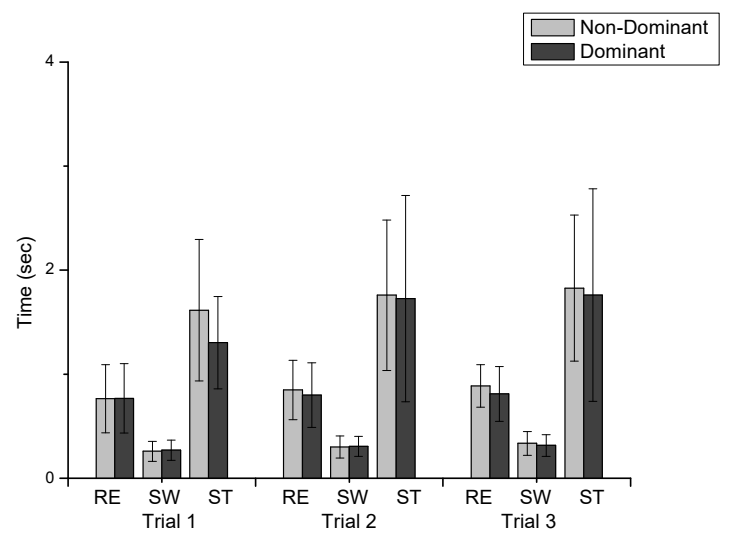

(B)

Figure 3. Preferred limb comparisons on reaction, swing, and step times between groups following three repeated perturbations. (A). The control group demonstrated significant differences on repetitions $(\mathrm{F}=24.06, p=0.001)$ and times $(\mathrm{F}=97.04, p=0.001)$. (B). The LBP group demonstrated a significant interaction on repetitions and times $(\mathrm{F}=6.03, p=0.02)$, and the times were significantly different $(\mathrm{F}=45.04, p=0.001)$. RE: reaction time, SW: swing time, ST: step time, the bar indicates standard deviation.

\section{Discussion}

The altered motions following perturbations led subjects with LBP to adopt compromised balance strategies. It was generally accepted that the LBP group was able to adjust their dynamic balance. Our results supported the first trial effect, which was studied utilizing lower trip amounts to benefit those older adults who could not tolerate larger perturbations [21]. Although results were limited to the LBP group, the repeated trip exposure was effective in a rapid adaptation to trips across functional activities [18]. The step time results could be explained by similar postural reactions; however, the results of their study were limited due to the inclusion of elderly participants without considering limb dominance.

The first hypothesis in our study was that individuals with LBP would demonstrate longer limb reaction and recovery step times following repeated trip perturbations. However, we rejected the hypothesis since the groups demonstrated a significant interaction on repetitions and times following repeated trip perturbations. The novelty of the first perturbation enhanced a protective strategy implemented by the LBP group, and they demonstrated longer recovery step times in subsequent perturbations.

Our results indicated that a protective strategy for recovery step time was adopted to avoid possible injuries following the first perturbation. The LBP group demonstrated postural control that was compromised during repeated perturbations. Their adaptive strategy could be used to prevent possible fall injuries. The co-contraction of the lower limb muscles could be explained as longer delays to the mechanical disturbance, which may cause a smaller angular deviation of the trunk [22,23]. As lower limb function involves postural stability, our study utilized the preferential side to better discriminate the behavior on limb preference [24].

A compensatory strategy is related to control of postural sway and trunk motions due to dual tasks in the LBP group [25]. It is expected, then, that the enhanced recovery step times in our study reflected stance time adjustments acting as a strategy against further injury or recurrent pain. The LBP group in our study was able to adjust for their balance deficits by developing longer step times to increase postural stability following repeated perturbations. It was also indicated that foot placement used to improve gait stability was altered to control foot placement as their traditional view of human locomotion [26]. Our results demonstrating the shorter step time could be utilized to alternative strategies for gait control and changes in body angular momentum. 
Our second hypothesis was that the limb reaction time delays were associated with the swing times following the first trip perturbation in the LBP group. We accepted this hypothesis as the LBP group demonstrated positive fair to moderate relationships based on the Pearson correlation coefficients, which ranged from 0.41 to 0.67 , while the control group's coefficients ranged from 0.23 to 0.57 . Although limb preference and dominance did not demonstrate a significant difference between groups, first limb reaction times and swing times demonstrated positive associations in the LBP group. The LBP group demonstrated that their limb reaction and swing times were significantly correlated following the repeated perturbations. The longer recovery swing times on their limbs could be minimized to avoid fall injuries following perturbations.

Our results indicated that the first limb reaction time did not demonstrate significant correlations with recovery step times in both groups, and the repeated reaction times demonstrated positive correlations. Although these studies reported an asymmetrical gait pattern in the stance phase of the LBP group, our results indicated reduced recovery step time on their preferred limb at the first perturbation, which led to potential injury. However, the control group demonstrated freedom of movement with pain as relatively slower COM displacement occurred from the COP. The shorter recovery step time in the LBP group indicated that their shorter response from the first perturbation would allow for less time for the COM displacement due to LBP. This control of foot placement in the LBP group was adjusted to ensure sufficient dynamic margins of stability relative to the trunk following the first novel perturbation.

We also expected a trunk stiffening strategy might occur because of reduced trunk motion (possibly due to pain). These patterns are likely to be functional to enhance postural stability in the LBP group [23]. However, the somatosensory integration for the control of the preferred foot placement in subsequent trials was based on swing and step times in the initial trial in order to enhance gait stability. Although reaction time was not significantly different between groups following the first perturbation, the longer recovery step times from repeated trips were similar in both groups. One study supported that the LBP group demonstrated reduced lumbar motion and increased double limb support time during stair climbing for balance stability [27]. However, their postural sway might not be directly related to reduced spine motion, but might possibly be linked to an increase in muscular active tension in the low back [28]. Although these studies did not carefully consider a trunk stiffening strategy relative to recovery step time, the LBP group may compromise their trunk and ankle control during repeated trips to adjust dynamic stability.

There were several limitations to this study. First, the subject variations or potential selection bias might be including demographic backgrounds between groups. Still, the power of the study was confirmed by the sample size as well as the standardized procedures used to control subject characteristics between groups. The LBP group completed selfassessments using the ODI questionnaire, and their moderate level of disability results were subjective. Second, our study did not focus on dynamic balance deficits. The foot placement measures following trip perturbations may not explain a cause-and-effect relationship due to the nature of the study design, which indicates observed relationships rather than causal effects.

\section{Conclusions}

The LBP group demonstrated reduced step time in response to a novel trip; however, their step times increased following repeated trips, and this adaptive postural strategy might be beneficial to avoid possible fall injuries. This protective strategy from repeated trips with optimal foot placements could be critical for rehabilitation strategies. Future studies are warranted to consider controlling foot placement and joint kinematics to ensure dynamic stability in the lower limbs following perturbations.

Author Contributions: Conceptualization, P.S.S.; methodology, P.S.S.; validation, E.H. and P.S.S.; formal analysis, E.H. and P.S.S.; investigation, E.H. and P.S.S.; resources, E.H. and P.S.S.; data curation, P.S.S.; writing—original draft preparation, P.S.S.; writing—review and editing, E.H. and 
P.S.S.; visualization, E.H.; supervision, P.S.S.; project administration, P.S.S. All authors have read and agreed to the published version of the manuscript.

Funding: This work was supported by the Herbert H. and Grace A. Dow College of Health Professions at Central Michigan University (ION 42041-15647 and FRCE 48702).

Institutional Review Board Statement: The study was conducted according to the guidelines of the Declaration of Helsinki and approved by the Institutional Review Board of Central Michigan University (protocol code: 955053-3; date of approval: 16 November 2016).

Informed Consent Statement: Informed consent was obtained from all subjects involved in the study.

Data Availability Statement: The data presented in this study are available on request from the corresponding author.

Conflicts of Interest: None of the authors have any financial or personal conflicts of interest in relation to the submission, other people, or any organizations.

\section{References}

1. Sung, P.S.; Leininger, P.M. A kinematic and kinetic analysis of spinal region in subjects with and without recurrent low back pain during one leg standing. Clin. Biomech. 2015, 30, 696-702. [CrossRef] [PubMed]

2. Tsao, H.; Galea, M.; Hodges, P. Reorganization of the motor cortex is associated with postural control deficits in recurrent low back pain. Brain 2008, 131, 2161-2171. [CrossRef] [PubMed]

3. Brumagne, S.; Janssens, L.; Janssens, E.; Goddyn, L. Altered postural control in anticipation of postural instability in persons with recurrent low back pain. Gait Posture 2008, 28, 657-662. [CrossRef] [PubMed]

4. Bruijn, S.M.; van Dieen, J.H. Control of human gait stability through foot placement. J. R. Soc. Interface 2018, 15, 20170816. [CrossRef]

5. Buurke, T.J.W.; Lamoth, C.; Vervoort, D.; van der Woude, L.; Otter, R.D. Adaptive control of dynamic balance in human gait on a split-belt treadmill. J. Exp. Biol. 2018, 221, jeb174896. [CrossRef] [PubMed]

6. Sung, P.S.; Cavataio, M.; Sauve, J. Adaptive trunk sway velocities following repeated perturbations in subjects with and without low back pain. J. Electromyogr. Kinesiol. 2020, 52, 102423. [CrossRef] [PubMed]

7. Mueller, J.; Engel, T.; Mueller, S.; Stoll, J.; Baur, H.; Mayer, F. Effects of sudden walking perturbations on neuromuscular reflex activity and three-dimensional motion of the trunk in healthy controls and back pain symptomatic subjects. PLoS ONE 2017, 12, e0174034. [CrossRef]

8. Beurskens, R.; Bock, O. Does the walking task matter? Influence of different walking conditions on dual-task performances in young and older persons. Hum. Mov. Sci. 2013, 32, 1456-1466. [CrossRef] [PubMed]

9. Mok, N.W.; Brauer, S.G.; Hodges, P.W. Failure to Use Movement in Postural Strategies Leads to Increased Spinal Displacement in Low Back Pain. Spine 2007, 32, E537-E543. [CrossRef]

10. Brumagne, S.; Janssens, L.; Knapen, S.; Claeys, K.; Suuden-Johanson, E. Persons with recurrent low back pain exhibit a rigid postural control strategy. Eur. Spine J. 2008, 17, 1177-1184. [CrossRef] [PubMed]

11. Simonsen, E.B. Contributions to the understanding of gait control. Dan. Med J. 2014, 61, B4823.

12. Musker, M.; Short, C.; Licinio, J.; Wong, M.-L.; Bidargaddi, N. Using behaviour change theory to inform an innovative digital recruitment strategy in a mental health research setting. J. Psychiatr. Res. 2019, 120, 1-13. [CrossRef]

13. Sadeghi, H.; Allard, P.; Prince, F.; Labelle, H. Symmetry and limb dominance in able-bodied gait: A review. Gait Posture 2000, 12, 34-45. [CrossRef]

14. Oldfield, R. The assessment and analysis of handedness: The Edinburgh inventory. Neuropsychologia 1971, 9, 97-113. [CrossRef]

15. Ciccone, D.S.; Just, N.; Bandilla, E.B. Non-organic symptom reporting in patients with chronic non-malignant pain. Pain 1996, 68, 329-341. [CrossRef]

16. Fairbank, J.C.; Pynsent, P.B. The Oswestry Disability Index. Spine 2000, 25, 2940-2953. [CrossRef]

17. Fairbank, J.C.; Couper, J.; Davies, J.B.; O’Brien, J.P. The Oswestry low back pain disability questionnaire. Physiotherapy 1980, 66, 271-273.

18. Pai, Y.-C.; Bhatt, T.; Wang, E.; Espy, D.; Pavol, M.J. Inoculation Against Falls: Rapid Adaptation by Young and Older Adults to Slips During Daily Activities. Arch. Phys. Med. Rehabil. 2010, 91, 452-459. [CrossRef] [PubMed]

19. Yang, F.; Kim, J.; Yang, F. Effects of obesity on dynamic stability control during recovery from a treadmill-induced slip among young adults. J. Biomech. 2017, 53, 148-153. [CrossRef]

20. Muller, K.E.; Barton, C.N. Approximate Power for Repeated-Measures ANOVA Lacking Sphericity. J. Am. Stat. Assoc. 1989, 84, 549-555. [CrossRef]

21. Pai, Y.-C.; Yang, F.; Bhatt, T.; Wang, E. Learning from laboratory-induced falling: Long-term motor retention among older adults. AGE 2014, 36, 1367-1376. [CrossRef]

22. Maaswinkel, E.; Griffioen, M.; Perez, R.; van Dieën, J. Methods for assessment of trunk stabilization, a systematic review. J. Electromyogr. Kinesiol. 2016, 26, 18-35. [CrossRef] 
23. Van Dieen, J.H.; Cholewicki, J.; Radebold, A. Trunk muscle recruitment patterns in patients with low back pain enhance the stability of the lumbar spine. Spine 2003, 28, 834-841. [CrossRef] [PubMed]

24. Carpes, F.P.; Diefenthaeler, F.; Bini, R.R.; Stefanyshyn, D.; Faria, I.E.; Mota, C.B. Does leg preference affect muscle activation and efficiency? J. Electromyogr. Kinesiol. 2010, 20, 1230-1236. [CrossRef]

25. Van Daele, U.; Hagman, F.; Truijen, S.; Vorlat, P.; Van Gheluwe, B.; Vaes, P. Decrease in postural sway and trunk stiffness during cognitive dual-task in nonspecific chronic low back pain patients, performance compared to healthy control subjects. Spine 2010, 35, 583-589. [CrossRef] [PubMed]

26. Artoni, F.; Fanciullacci, C.; Bertolucci, F.; Panarese, A.; Makeig, S.; Micera, S.; Chisari, C. Unidirectional brain to muscle connectivity reveals motor cortex control of leg muscles during stereotyped walking. NeuroImage 2017, 159, 403-416. [CrossRef] [PubMed]

27. Hicks, G.E.; Sions, J.M.; Coyle, P.C.; Pohlig, R.T. Altered spatiotemporal characteristics of gait in older adults with chronic low back pain. Gait Posture 2017, 55, 172-176. [CrossRef]

28. Hamaoui, A.; Do, M.; Bouisset, S. Postural sway increase in low back pain subjects is not related to reduced spine range of motion. Neurosci. Lett. 2004, 357, 135-138. [CrossRef] 